\title{
Neutron radiative capture reactions on nuclei of relevance to $0 v \beta \beta$, dark matter and neutrino/antineutrino searches
}

\author{
W. Tornow ${ }^{1,2, a}$ and Megha Bhike ${ }^{1,2}$ \\ ${ }^{1}$ Department of Physics, Duke University, Durham, North Carolina 27708, USA \\ ${ }^{2}$ Triangle Universities Nuclear Laboratory, Durham, North Carolina 27708, USA
}

\begin{abstract}
A program is underway at the Triangle Universities Nuclear Laboratory (TUNL) to measure the neutron capture cross section in the 0.5 to $15 \mathrm{MeV}$ energy range on nuclei whose radioactive daughters could potentially create backgrounds in searches for rare events. Here, we refer to neutrino-less double-beta decay and dark-matter searches, and to detectors built for neutrino and/or antineutrino studies. Neutron capture cross-section data obtained by using the activation method are reported for ${ }^{40} \mathrm{Ar}{ }^{74,76} \mathrm{Ge},{ }^{128,130} \mathrm{Te}$ and ${ }^{136} \mathrm{Xe}$ and compared to model calculations and

evaluations.
\end{abstract}

\section{Introduction}

Although most experiments aimed at detecting rare events are mounted deep underground in mines, cosmicray produced spallation neutrons and neutrons from $(\alpha, n)$ reactions are a major concern. Spallation neutrons undergo a variety of reactions during the slowing-down process. Unless scattering takes place on hydrogen or other light nuclei, neutron elastic scattering is not an efficient way of slowing down neutrons. The strongly forward-peaked cross section typical for scattering of medium mass nuclei (like in rocks) does not result in substantial energy losses and trajectory changes. Therefore, inelastic scattering and neutron-induced reactions are the main process for degrading the energy spectrum and changing the initial trajectory of spallation neutrons. Once the neutron energy reaches values below $50 \mathrm{MeV},(\mathrm{n}, 4 \mathrm{n})$ and $(\mathrm{n}, 3 \mathrm{n})$ and $(\mathrm{n}, 2 \mathrm{n})$ reactions with their large cross sections (of the order of barns) are the dominant contributor to the neutron slowing-down process. The associated multiplication of the neutron flux is often overlooked in estimates of the neutron flux from the measured muon flux at a given depth in the vicinity of a detector. These $(\mathrm{n}, \mathrm{xn})$ reactions are the most powerful process in slowing down medium energy neutrons until their energy approaches approximately $8 \mathrm{MeV}$. The resulting daughter nuclei are often radioactive and the decay $\gamma$ rays are a potential source of background in many rare event searches. Accidently, $8 \mathrm{MeV}$ is also the highest energy of $(\alpha, n)$ neutrons produced in rocks and shielding and detector materials. Here, the $\alpha$-particles originate from the uranium and thorium decay chains. In the neutron energy regime from $8 \mathrm{MeV}$ down to 3.5 $\mathrm{MeV}$, inelastic neutron scattering (i.e., (n, $\left.\mathrm{n}^{\prime} \gamma\right)$ reactions) and the radiative neutron capture $(n, \gamma)$ are the two most important sources of $\gamma$-ray background. Below $3.5 \mathrm{MeV}$ neutron energy (assuming that $2039 \mathrm{keV}$, the Q-value for the $0 v \beta \beta$ of ${ }^{76} \mathrm{Ge}$ is the lowest $\gamma$-ray energy of interest), the radiative neutron capture becomes the sole source of $\gamma$-ray background. In contrast to neutron inelastic scattering, the neutron capture process produces much higher energy $\gamma$ rays (up to the neutron binding energy plus the neutron kinetic energy) and often a cascade of de-excitation $\gamma$ rays. The recoil nuclei produced in neutron elastic and inelastic scattering and neutron capture processes are potential backgrounds in WIMP dark-matter searches. In the latter two cases, the associated $\gamma$ rays must escape the detection process for causing a misinterpretation as a potential WIMP event.

As has been stated above, the radiative neutron capture below $8 \mathrm{MeV}$ is an important source of potential $\gamma$-ray background in rare event searches, especially $0 v \beta \beta$ searches on ${ }^{76} \mathrm{Ge},{ }^{130} \mathrm{Te}$, and ${ }^{136} \mathrm{Xe}$. In the following we report on neutron capture cross-section measurements on these isotopes. In addition, we include ${ }^{40} \mathrm{Ar}$, because it is an important cooling and shielding material in the $0 v \beta \beta$ decay search of the GERDA [1] collaboration. Furthermore, in its own right as the detector medium for the currently designed Long Baseline Neutrino Experiment (LBNE) [2], neutron induced background reactions on ${ }^{40} \mathrm{Ar}$ are of great importance for neutrino physics.

\section{Experimental setup and procedure}

Monoenergetic neutrons are produced via the ${ }^{3} \mathrm{H}(\mathrm{p}, \mathrm{n})^{3} \mathrm{He}$ $\left(\mathrm{E}_{\mathrm{n}}=0.37\right.$ to $\left.3.6 \mathrm{MeV}\right),{ }^{7} \mathrm{Li}(\mathrm{p}, \mathrm{n})^{7} \mathrm{Be}\left(\mathrm{E}_{\mathrm{n}}=0.56 \mathrm{MeV}\right)$, ${ }^{2} \mathrm{H}(\mathrm{d}, \mathrm{n}){ }^{3} \mathrm{He}\left(\mathrm{E}_{\mathrm{n}}=4.5\right.$ to $\left.7.4 \mathrm{MeV}\right)$, and ${ }^{3} \mathrm{H}(\mathrm{d}, \mathrm{n}){ }^{4} \mathrm{He}$ $\left(\mathrm{E}_{\mathrm{n}}=14.8 \mathrm{MeV}\right)$ reactions. The charged-particle beams are

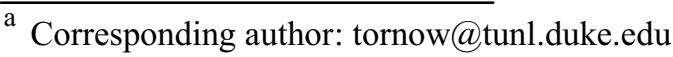


produced and accelerated at TUNL's tandem accelerator facility [3]. Typically, the proton and deuteron beam currents on target range between 2 and $4 \mu \mathrm{A}$, resulting in neutron fluxes of the order of $10^{7}$ per $\mathrm{cm}^{2}$ and $\mathrm{s}$ at the position of the sample of interest. The associated neutron energy spread increases from $\pm 100 \mathrm{keV}$ at the lowest energy to $\pm 330 \mathrm{keV}$ at $7.6 \mathrm{MeV}$, while at $14.8 \mathrm{MeV}$ the energy spread is only $\pm 50 \mathrm{keV}$. The argon and xenon gases are contained at high pressure $(\sim 150 \mathrm{~atm})$ in lowmass metal spheres [4]. Typical arrangements are shown in Fig. 1 for gaseous (top) and solid (bottom) samples.
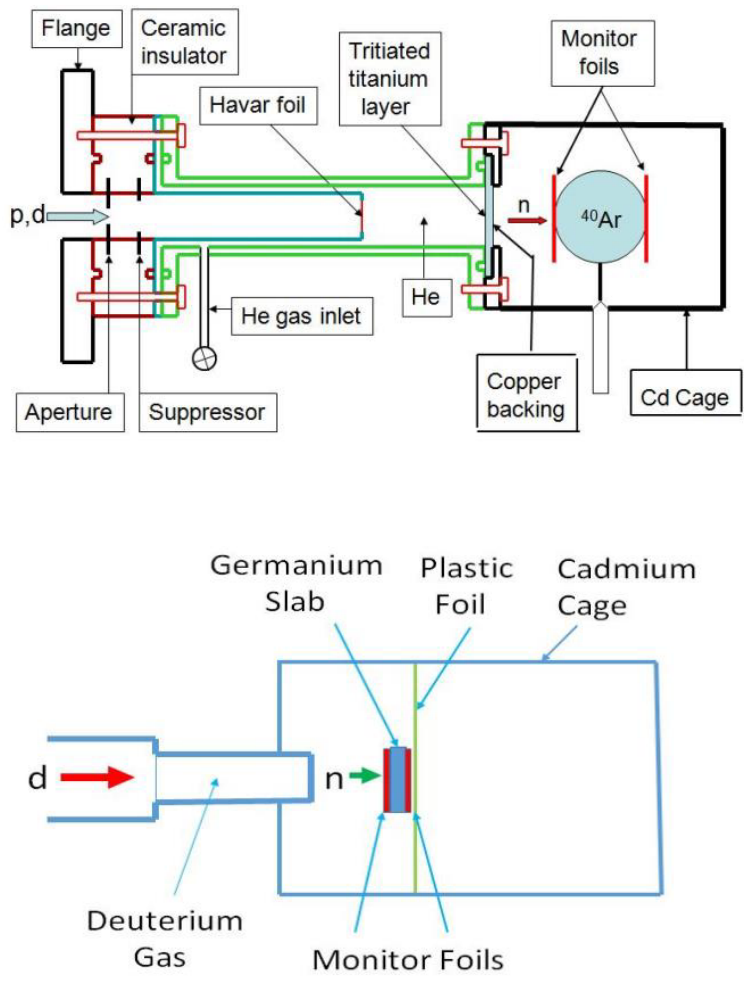

Figure 1. (top) Typical experimental setup using a tritiated target based neutron source and a gaseous sample contained in a high-pressure cell with neutron fluence monitor foils attached to. (bottom) Same as above using deuterium gas cell based neutron source and a solid slab germanium sample.

Titanium spheres of $10 \mathrm{~mm}$ diameter and $0.2 \mathrm{~mm}$ wall thickness or stainless-steel spheres of $20 \mathrm{~mm}$ diameter and $0.6 \mathrm{~mm}$ wall thickness are used. The metallic germanium samples enriched to $86 \%$ in ${ }^{76} \mathrm{Ge}$ and $14 \%$ in ${ }^{74} \mathrm{Ge}$, have an area of $10 \mathrm{~mm} \times 10 \mathrm{~mm}$ and thickness of $2 \mathrm{~mm}$. The tellurium metal powder of natural abundances $\left(34 \%{ }^{130} \mathrm{Te}\right.$ and $\left.32 \%{ }^{128} \mathrm{Te}\right)$ is contained in a plastic pill box of $12 \mathrm{~mm}$ diameter and $7 \mathrm{~mm}$ thickness. Thin indium or gold monitor foils are attached to the front and back side of the samples for neutron fluence determination. In order to prevent room-return thermal neutrons from capturing on the sample of interest, it is surrounded by an enclosure made of cadmium $(0.5 \mathrm{~mm}$ wall thickness).

Neutron irradiation times varied between 2 minutes and several hours, depending on the half-life time of the daughter nucleus produced after neutron capture. After irradiation, the samples and the monitor foils are $\gamma$-ray counted with well-shielded 60\% HPGe detectors of accurately known photo-peak efficiency.

The data-acquisition system consists of the Canberra Multiport II hardware running the GENIE software [5]. The intensity of the $\gamma$-ray lines of interest is followed over a time period of several half-life times, except for the $355.73 \mathrm{keV} \gamma$ ray of ${ }^{196} \mathrm{Au}$ with its long half-life time of $\mathrm{T}_{1 / 2}=6.167 \mathrm{~d}$. The accumulated $\gamma$-ray energy spectra were analysed using the software package TV [6].

The activation formula is used twice. First, for determining the neutron flux at the position of the sample using the data obtained from the monitor foils and the known cross section. Second, for obtaining the capture cross section for the sample of interest using the measured neutron fluence. The activation formula is given in Eq. (1).

$$
\sigma=\frac{A \lambda}{N \phi \varepsilon I_{\gamma}\left(1-e^{-\lambda t_{i}}\right) e^{-\lambda t_{d}\left(1-e^{-\lambda t_{m}}\right)}}
$$

Here, $\sigma$ is the reaction cross section, the activity A is the total yield in the photo-peak of the reaction of interest, $\mathrm{N}$ is the number of target nuclei, $\phi$ is the neutron flux per $\mathrm{cm}^{2}$ and $\mathrm{s}, \varepsilon$ and $\mathrm{I}_{\gamma}$ are the photo-peak efficiency and the branching ratio for the $\gamma$-ray of interest, respectively, and $\lambda$ denotes the decay constants of the nucleus involved. The times $t_{i}, t_{d}$, and $t_{m}$ are the irradiation time, decay time between the end of irradiation and the start of counting, and the measuring time, respectively.

The neutron flux at the position of the sample is not simply the average of the values obtained from the two monitor foils due to the tight geometry of the experimental setup. Monte-Carlo calculations are performed to obtain the effective neutron fluence, average neutron energy and its associated energy spread seen by the sample. Monte-Carlo techniques are also used to correct for finite-geometry effects associated with the $\gamma$-ray counting of the samples of interest, which differ in size from the $\gamma$-ray test sources used to measure the absolute efficiency of the HPGe detectors. Here, the attenuation of the $\gamma$ rays in the sample is corrected for as well.

\section{Results}

The measured neutron capture cross-section results obtained in the present work [7] for the reaction ${ }^{40} \mathrm{Ar}(\mathrm{n}, \gamma){ }^{41} \mathrm{Ar}$ are given in Fig. 2 together with the only existing previous results $[8,9]$. The TENDL-2013 calculation based on the TALYS code [10] gives an excellent description of the present data. Data in the 9 to $12 \mathrm{MeV}$ energy range are still needed to find out whether the ENDF/B-VII.1 evaluation [11] is really underestimating the cross section by as much as almost one order of magnitude at $11 \mathrm{MeV}$. The previously 
existing data (below about $1 \mathrm{MeV}$ ) are in very good agreement with our results.

Results for the ${ }^{76} \mathrm{Ge}(\mathrm{n}, \gamma){ }^{77} \mathrm{Ge}$ total cross section are shown in Fig. 3 [12] in comparison to the only previously existing datum at $14 \mathrm{MeV}$ [13], and the results of the model calculation TENDL-2013 and nuclear data evaluations. The data are important for the GERDA [1] and Majorana [15] collaborations. Again, the TALYS code provides a very good description of the present data, in close agreement with the ENDF/B-VII.1 evaluation.

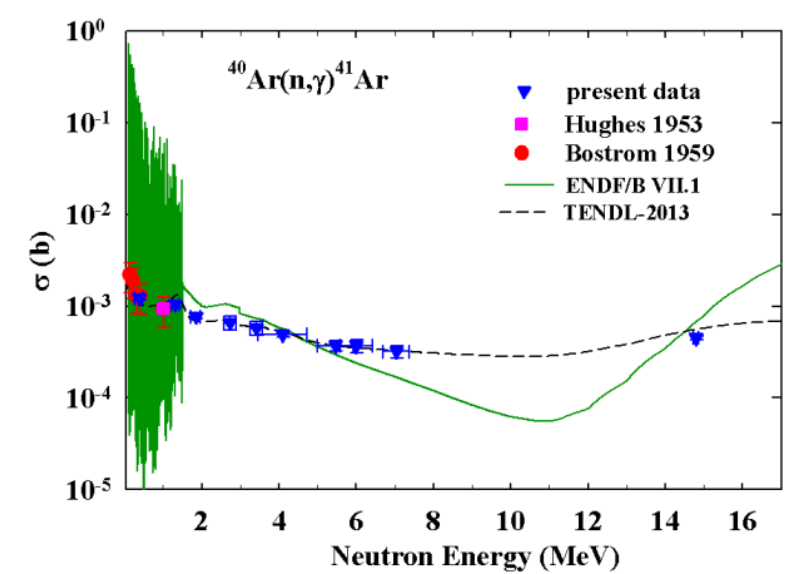

Figure 2. Results for ${ }^{40} \operatorname{Ar}(n, \gamma)^{41} \mathrm{Ar}$ radiative capture cross section in comparison to previous data (available only below 1 $\mathrm{MeV}$ ), and the TENDL-2013 prediction and ENDF/B-VII.1 evaluation.

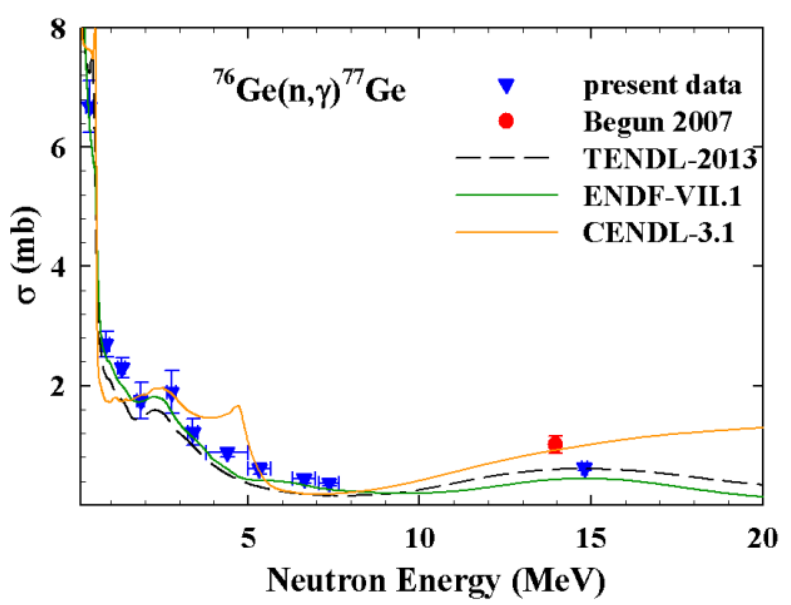

Figure 3. ${ }^{76} \mathrm{Ge}(\mathrm{n}, \gamma){ }^{77} \mathrm{Ge}$ total cross-section data in comparison to the previous datum at $14 \mathrm{MeV}$, the model calculation TENDL-2013 and the evaluations ENDF/B-VII.1 and CENDL3.1 [14].

Data for the neutron capture cross section to the isomeric state of ${ }^{77} \mathrm{Ge}$ at $159.7 \mathrm{keV}$ are also given in [12]. In contrast to ${ }^{76} \mathrm{Ge}$, neutron capture cross-section data on ${ }^{74} \mathrm{Ge}$ do exist in the literature up to almost $4 \mathrm{MeV}$. Our results are given in Fig. 4 in comparison to the previous data of Tolstikov et al. [16], Pasechnik et al. [17], and Trofimov [18]. Our capture cross-section data to the $139.7 \mathrm{keV}$ isomeric state in ${ }^{75} \mathrm{Ge}$ are given in [12]. Although the data-acquisition for neutron capture on the ${ }^{128} \mathrm{Te}$ and ${ }^{130} \mathrm{Te}$ isotopes has been completed, the data analysis is still underway. Therefore, final results will not be given here. Our data are important for the $0 v \beta \beta$ decay search of ${ }^{130} \mathrm{Te}$ performed by the CUORE collaboration [19]. Finally, our data for the reaction ${ }^{136} \mathrm{Xe}(\mathrm{n}, \gamma){ }^{137} \mathrm{Xe}$ are shown in Fig. 5 [20]. While the TENDL-2012 calculation gives larger cross-section values, the ENDF/B-VII evaluation agrees with our data up to about $3 \mathrm{MeV}$ and

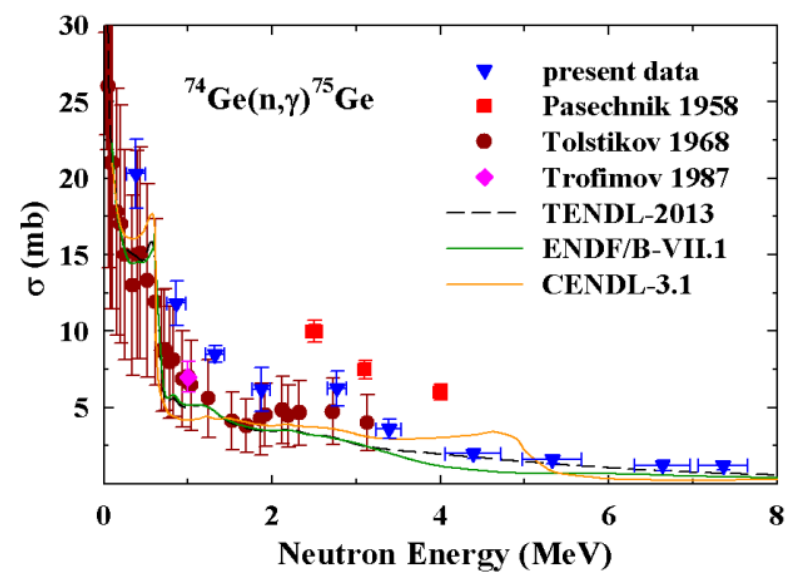

Figure 4. ${ }^{74} \mathrm{Ge}(\mathrm{n}, \gamma){ }^{75} \mathrm{Ge}$ total cross-section data in comparison to previously existing data (available below $4 \mathrm{MeV}$ ), the model calculation and evaluations referred to in the figure caption of Fig.3.

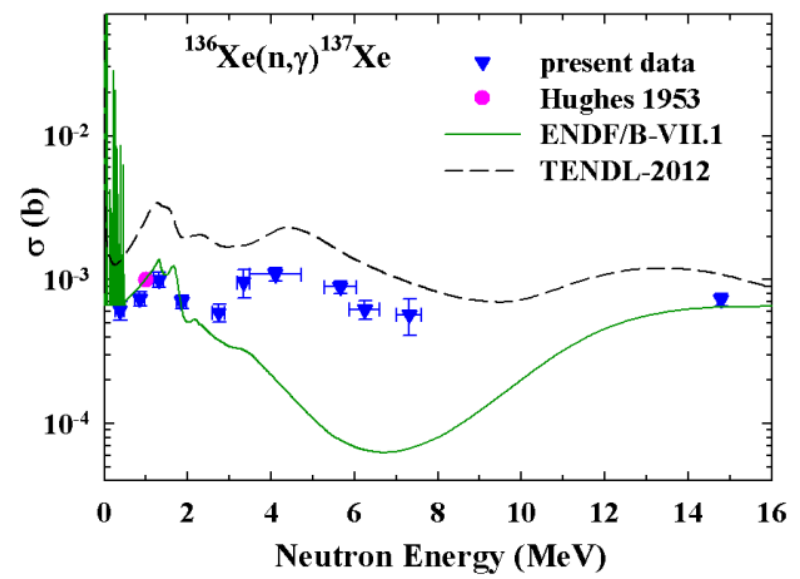

Figure 5. ${ }^{136} \mathrm{Xe}(\mathrm{n}, \gamma){ }^{137} \mathrm{Xe}$ radiative capture cross-section data in comparison to the model calculation TENDL-2012, the ENDF/B-VII.1 evaluation, and the previous datum at $1 \mathrm{MeV}$.

again at $14.8 \mathrm{MeV}$, but in between the evaluated crosssection data are approximately one order of a magnitude lower than the trend of the present data. The only previously existing datum of Hughes [8] at $1 \mathrm{MeV}$ agrees very well with our data, which are important for the $0 v \beta \beta$ decay searches of the EXO-200 [21] and KamLAND-Zen [22] collaborations.

\section{Conclusions}

The present data provide for the first time the basis for accurately calculating the neutron radiative-captureinduced background in $0 v \beta \beta$ searches, once the neutron flux and energy distribution at the location of the 
experimental setup is known. The ${ }^{40} \operatorname{Ar}(\mathrm{n}, \gamma)^{41} \mathrm{Ar}$ data are important for neutrino detectors based on liquid argon technology, like LBNE. Furthermore, using liquid argon or HPGe $\gamma$-ray detectors, the present data in conjunction with the TALYS based model calculation TENDL-2013 could be used to determine the integrated neutron flux at any detector site of interest, assuming a reasonable neutron energy distribution.

\section{Acknowledgements}

The authors acknowledge valuable contributions from A. P. Tonchev, LLNL, S.W. Finch and Krishichayan, Duke University, and M. E. Gooden, North Carolina State University. This work was supported in part by the US Department of Energy, Office of Nuclear Physics, under Grant No. DE-FG02-97ER41033.

\section{References}

1. M. Agostini et al., Phy. Rev. Lett. 111, 122503 (2013)

2. http://lbne.fnal.gov/

3. http://www.tunl.duke.edu/

4. G. Rupp et al., Nucl. Instrum Methods Phys. Res. A 608, 152 (2009)

5. http://www.canberra.com

6 J. Theuerkauf et al., Institute of Nuclear Physics, University of Cologne (1993)

7. Megha Bhike, B. Fallin, and W. Tornow, Phys. Lett. B736, 361 (2014)

8. D. J. Hughes, R. C. Garth, and J. S. Levin, Phys. Rev. 91, 1423 (1953)

9. N. A. Bostrom, I. L. Morgan, J. T. Prud'homme, P. L. Okhuyaen, O. M. Hudson Jr., Wright Air Development Centre Report TN-59-107, USA, 1959.

10. A. J. Koning and, D. Rochman, Nucl. Data Sheets 113, 2841 (2012)

11. M. B. Chadwick et al., Nucl. Data 112, 2887 (2011).

12. Megha Bhike, B. Fallin, Krishichayan, and W. Tornow, submitted for publication.

13 S. V. Begun, O. G. Druzheruchenko, O. O. Puripirina, and V. V. Tarakanaov, http://arxiv.org/abs/nucl-ex/0701039

14. Z. G. Ge et al., Proc. Int. Conf. on Nucl. Data for Sci. and Technol., Jeju Island, Korea, p26 (2010)

15. N. Abgrall et al., Advances in High Energy Physics, 2014, 365432 (2014)

16. V. A. Tolstikov, V. K. P. Koroleva, V. E. Kolesov, and A. G. Dovbenko, Atomnya Energiya 23, 566 (1967)

17. M. V. Pasechnik et al., Int. At. Ener. Conf. Vol 15, p. 18 (1958)

18. Yu. N. Torfimov, Int. Conf. On Neutron Physics. Kiev, 14-18 Sept. 1987, vol. 3, p. 331

19. www.nu.to.infn.it/exp/all/cuore/

20. Megha Bhike and W. Tornow, Phys. Rev. C89, 031602 (2014)

21. J. B. Albert et al., Nature 510, 229 (2014)

22. A. Gando et al., Phys. Rev. Lett. 110, 062502 (2013) 